\title{
Large-scale conversion of helical-ribbon carbon nanofibers to a variety of graphene-related materials
}

B. Lobato, ${ }^{a}$ C. Merino, ${ }^{b}$ V. Barranco ${ }^{c}$ and T.A. Centeno ${ }^{a}{ }^{*}$

a Instituto Nacional del Carbón-CSIC, Apartado 73, 33080 Oviedo, Spain.Email:teresa@incar.csic.es; Fax:+34.985297662; Tel: +34.985119090

${ }^{b}$ GRAnPH Nanotech. Grupo Antolín Ingeniería, Ctra. Madrid - Irún, km. 244.8, Burgos, Spain.

${ }^{c}$ Instituto de Ciencia de Materiales de Madrid-CSIC, Sor Juana Inés de la Cruz 3, Cantoblanco, 28049 Madrid, Spain.

Helical-ribbon carbon nanofibers produced at industrial scale were successfully converted in highly functionalized graphene nanoplatelets by using a slight modification of the Hummers oxidation method. The duration of the oxidative process severely affected the interlayer spacing in the resulting nanoplatelets and, consequently, they showed very different exfoliation behavior. Therefore, it was possible to obtain a variety of graphene-related products through their ultrasonication or thermal treatments such as exfoliation-reduction by flash-pyrolysis in air at temperatures between 400 and $1000^{\circ} \mathrm{C}$ or standard activation with $\mathrm{CO} 2$ at $800^{\circ} \mathrm{C}$. Detailed comparison of the functionalized carbon nanoplatelets, graphene oxides, reduced graphene oxides and activated carbon nanoplatelets reveals the wide spectrum of their properties with specific surface areas in the range $4-500 \mathrm{~m}^{2} / \mathrm{g}$, oxygen content from 38 to $5 \mathrm{wt} . \%$ and different structural ordering. This study also underlines the impact of the structural, textural and chemical changes experienced by the carbon nanofibers along the various processes on the performance as supercapacitor electrodes. This preliminary study, based on cyclic voltammetry in $2 \mathrm{M} \mathrm{H}_{2} \mathrm{SO} 4$ aqueous electrolyte, becomes a summary of the strengths and weaknesses of the different graphene-related materials for this application. Whereas the helical-ribbon carbon nanofibers displayed only $10 \mathrm{~F} / \mathrm{g}$, the capacitance of the functionalized graphene nanoplatelets greatly rose to $104 \mathrm{~F} / \mathrm{g}$ with clear contributions from pseudocapacitance. Values around 100-120 F/g were found for graphene oxides and activated graphene nanoplatelets although a marked resistive character is detected. Flash-pyrolysis at $1000^{\circ} \mathrm{C}$ leads to lower capacitance $(79 \mathrm{~F} / \mathrm{g})$ but much quicker charge propagation. Among all these materials, the lower-cost functionalized graphene nanoplatelets displayed the better behavior for aqueous supercapacitor. 


\section{Introduction}

The unique properties of graphene have raised great interest for its application in numerous fields ranging from energy storage, biomedical uses, composites, functional coatings to electronic devices, etc. ${ }^{1-5}$ Among the wide variety of methods proposed to obtain graphene, ${ }^{1}$ the top-down method based on the exfoliation of graphite is the most commonly used for mass production. This can be achieved through i) direct liquid exfoliation by shearing and sonication, ii) intercalation of electrolyte ions and electrochemical initiated-exfoliation or iii)

by intercalating functionalities between the carbon layers to weaken their interaction followed by diverse exfoliation processes. ${ }^{6,7}$

Current research efforts are mainly focused on the last route in view of its high yield, potential scalability and excellent processability of the resulting materials. It is commonly performed by harsh oxidation of graphite in acid media and subsequent ultrasonication ${ }^{3,8,9}$ to generate graphene oxide nanoplatelets. A controlled reduction leads finally to reduced graphene oxide. ${ }^{3,9}$ Such a method is simple but a high amount of non-exfoliated graphite still remains. Further studies have reported that oxidative methods may result more efficient on materials formed by a smaller number of carbon layers sheets such as MWCNTs $^{10,11}$ and carbon fibers. ${ }^{12}$

Twisted ribbon-shaped carbon nanofibers (GANF®) comprise a multilayer ribbon of 5-6 stacked graphene layers rolled up along the fiber axis forming a continuous spiral. ${ }^{13-15}$ Such a configuration facilitates interlaminar accessibility if compared to natural graphite and $\mathrm{MWCNTs}^{12}$ and the helical ribbon is successfully unraveled and cut by strong oxidation and mild sonication. It has been found that the resulting graphene oxide nanoplatelets lead to impressive improvements in the fracture toughness and the fatigue life of the GO/epoxy nanocomposites. ${ }^{16}$

We now report a further study on the potential of these singular carbon nanofibers, produced at industrial scale by the floating catalyst method in a continuous CVD process, as precursor of graphene materials. It is shown that the duration of the oxidation severely affects the interlayer spacing in the resulting functionalized graphene nanoplatelets (OFs) and, consequently, their intrinsic properties. Furthermore, the availability of OFs with different spacing 
makes possible to obtain a variety of graphene products by ultrasonication, exfoliation-reduction by flash-pyrolysis in air or by standard activation with $\mathrm{CO}_{2}$. This is an attractive approach toward a fabrication route of graphene-related materials at industrial scale. The clear distinction between functionalized graphene nanoplatelets, graphene oxides, reduced graphene oxides and activated graphene nanoplatelets with a wide features spectrum suggests their potential towards many applications.

Currently, supercapacitors (SC) are under an increasing demand as alternative energy storage devices. They are advanced systems where the electrical energy is physically stored by electrostatic adsorption of electrolytes ions on conductive electrodes. They are capable of storing and providing high amount of electrical energy in very short periods of time and display extremely long cycling-life and low maintenance cost. These features hold application for powering electric vehicles, portable electronic devices, uninterruptable power supplies, etc. ${ }^{17,18}$

A wide variety of carbons have been extensively investigated as electrodes in $\mathrm{SC}^{17,18}$ but the unique set of properties of graphene such as an extremely large theoretical surface area and outstanding electrical conductivity makes it the most promising candidate to improve energy density without sacrificing power. ${ }^{3,4,19,20}$ However, systematic studies have pointed out that the method by which graphene is synthesized defines its electrochemical properties, the surface charge and hydrophobicity. ${ }^{5,21}$ In fact, functional groups, structural defects, presence of metal oxides or surfactants impurities, residual moieties, arrangement of graphene-type sheets, etc. notably interfere towards the supercapacitors performance. ${ }^{22-25}$

As a first attempt, the possibilities of the variety of products produced by this method as SC electrodes have been briefly examined. The absence of surfactants in the synthesis allows getting reliable information of the impact of the structural, textural and chemical changes along the various processes on the electrochemical response of the resulting graphene materials. 


\section{Experimental}

\section{Materials synthesis.}

Helical-ribbon carbon nanofibers (GANF® grade D\&S) manufactured by Grupo Antolín Ingeniería (Burgos, Spain) ${ }^{13-15}$ using natural gas and a sulphide feedstock at over $1100^{\circ} \mathrm{C}$ by the floating catalyst technique were used as raw material.

Fig. 1 schematically summarizes the diverse treatments carried out for the synthesis of the different materials.

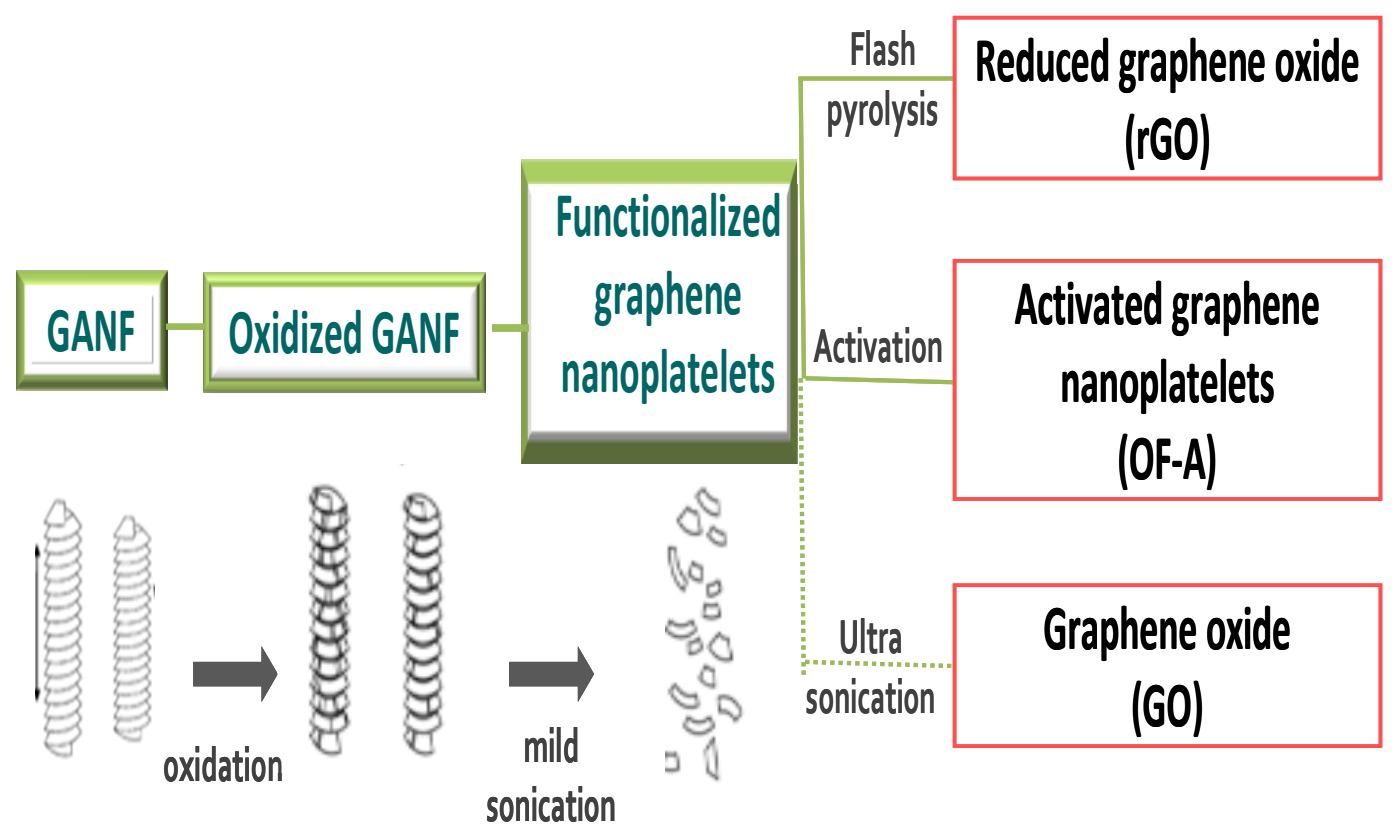

Fig. 1 Scheme of the materials synthesis.

Wet oxidation of carbon nanofibers. Functionalized carbon nanoplatelets were prepared from GANF by using a slight modification ${ }^{10}$ of the Hummers oxidation method. ${ }^{26}$ To achieve different oxidized materials, in the first oxidation step, the reactive solution $\left(\mathrm{NaNO}_{3} / \mathrm{H}_{2} \mathrm{SO}_{4} / \mathrm{KMnO}_{4} / \mathrm{GANF}\right)$ was stirred at $35^{\circ} \mathrm{C}^{12}$ for $150 \mathrm{~min}$ (Oxidation 1) or overnight (Oxidation 2). A subsequent mild mechanical exfoliation was carried out in water or other polar solvents by tip sonication. ${ }^{12}$ The resulting functionalized graphene nanoplatelets were labeled as OF1 and OF2, respectively.

Flash-heating. It was performed in air by rapid insertion of OF1 and OF2 into hot furnace at temperatures ranging from $400^{\circ} \mathrm{C}$ to $1000^{\circ} \mathrm{C}$. The resulting 
reduced graphene oxides were designated as rGO1-X and $\mathrm{rGO}-\mathrm{X}$ which indicates that the materials were prepared from OF1 and OF2 at temperature $X$. Activation. In a typical process, OF1 and OF2 were heated under $\mathrm{CO}_{2}$ at $20^{\circ} \mathrm{C} / \mathrm{min}$ up to $800^{\circ} \mathrm{C}$ and 2 hours soaking, resulting samples OF1-A and OF2A, respectively.

Further sonication of the highly oxidized nanoplatelets. Following the standard procedure to obtain graphene oxide, GO1 and GO2 were prepared, respectively, by ultrasonication of OF1 and OF2 in an ultrasound bath cleaner (J.P.Selecta Ultrasons system, $50-60 \mathrm{kHz}$ ) for $4 \mathrm{~h}$.

\section{Materials characterization}

A variety of techniques was used to monitor the chemical and structural changes occurring in the different materials.

A LECO Tru Spec microanalyzer was used to calculate the $(C, H, N)$ composition of the oxidized carbon fibers. The oxygen content was directly directly in a LECO-TF-900 furnace coupled to a LECO-CHNS-932 microanalyzer. The accuracy was around $\pm 5 \%$.

The chemical characterization of the samples was completed by the analysis of the surface chemistry by X-ray photoelectron spectroscopy (XPS). For the measurements, a customized SPECS system was employed working under a pressure of $5 \cdot 10^{-9}$ mbar with a MgKa X-ray source operated at $150 \mathrm{~W}$. The XPS high-resolution spectra were analyzed by CasaXPS software. In order to identify the functional groups and their respective percentages, C1s spectra were deconvoluted by applying Gaussian-Lorentzian (80:20) functions, after Shirley background correction.

The morphology of the samples was examined by Scanning Electron Microscopy (SEM) on a QUANTAN FEG 650, FEI equipment operating at $5 \mathrm{kV}$ and by Transmission Electron Microscopy (TEM) 2000EX-II (JEOL) instrument, using acceleration voltage of $160 \mathrm{kV}$.

The structural characterization involved X-Ray Diffraction by a D5000 (Siemens) instrument operating at $40 \mathrm{kV}$ and $40 \mathrm{~mA}$ and employing CuKa radiation, $\lambda=1.5406 \AA$ (The data were collected at steps of $0.02^{\circ}$ and intervals of $1 \mathrm{~s}$ per step in the $2 \theta$ range of $5-90^{\circ}$ ) and Raman Spectroscopy by means of a high-resolution LabRam HR UV800 (JYV-Jobin Yvon) spectrometer coupled 
to confocal microscope BXFM-ILHS (Olympus). A CDPS532M (JSDU) argon DPSS laser was employed at $\lambda=532 \mathrm{~nm}$, with a spot size of $3-5 \mu \mathrm{m}$ and output power at $24.3 \mathrm{~mW}$ (The spectra were recorded from 800 to $3500 \mathrm{~cm}^{-1}$ ).

The textural features were estimated from the analysis of nitrogen sorption isotherms at 77K (Micromeritics ASAP 2010) by different methods such as BET equation, Dubinin's theory, DFT approach and comparison plot. $^{27,28}$ The experimental error in the determination of surface area has been estimated to be around $\pm 10 \%$

\section{Electrochemical measurements}

The behavior of the various materials as supercapacitor electrodes was studied in a two-electrode system with two carbon pellets ( $8 \mathrm{~mm}$ in diameter) separated by glassy fibrous paper (300 $\mu \mathrm{m}$ thick) and placed in a Swagelok cell. The electrodes were prepared by mixing $90 \mathrm{wt} \%$ of the carbon powder with $5 \mathrm{wt} \%$ of polytetrafluoroethylene (PTFE) and $5 \mathrm{wt} \%$ of carbon black (Super-P). Cyclic voltammetry $(\mathrm{CV})$ tests at various scan rates $(1-50 \mathrm{mV} / \mathrm{s})$ were performed between 0 and $0.8 \mathrm{~V}$ in $2 \mathrm{M} \mathrm{H}_{2} \mathrm{SO}_{4}$ aqueous electrolyte. The specific capacitance of the electrode was calculated according to

$C=\left[q_{\mathrm{a}}+\left|q_{\mathrm{c}}\right|\right] / m_{\mathrm{c}} V$, where $q_{\mathrm{a}}$ and $q_{\mathrm{c}}$ are the anodic and cathodic voltammetric charges on positive and negative sweeps, $V$ is the potential range and $m_{\mathrm{c}}$ is the weight of carbon loaded in the composite electrode. ${ }^{29}$ Galvanostatic chargedischarge cycles at current densities between 1 and $100 \mathrm{~mA} / \mathrm{cm}^{2}$ (not shown) were used to cross-check (Table S2).

\section{Results and discussion}

\section{Characteristics of the graphene-related materials}

At first glance, oxidation processes (1) and (2) appear to be equally successful. The Raman spectra for the resulting samples yield virtually identical traces (Fig. 2a). The relative intensity $I_{D} / l_{G}$ notably varies from 0.03 for the pristine nanofibers up to 0.96 and 0.86 for OF1 and OF2, revealing the reduction of the in-plane $\mathrm{sp}^{2}$ domains induced by the introduction of defects and disorder. 

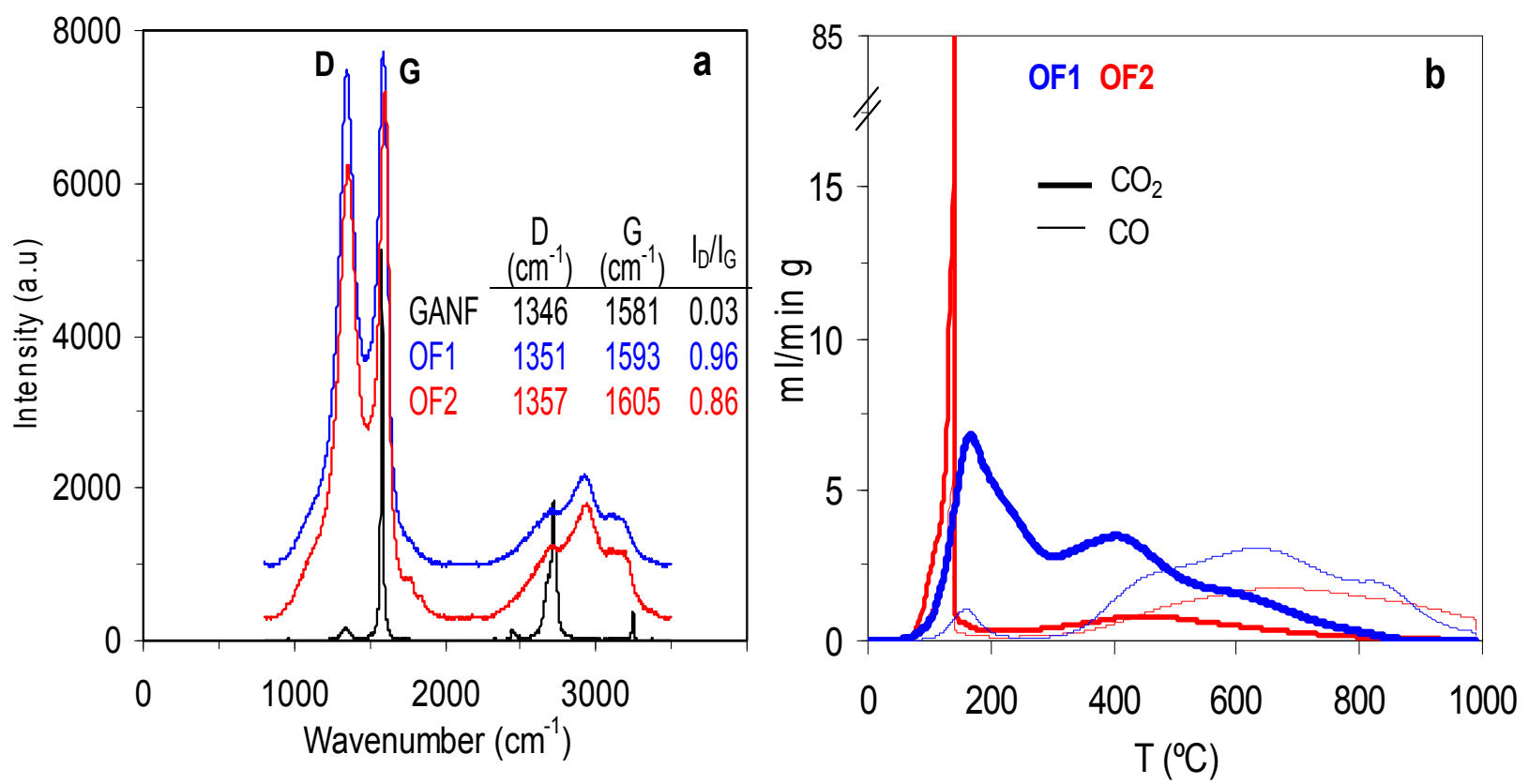

Fig. 2 Raman spectra (a) and TPD profiles (b) for carbon nanofibers and oxygen-rich graphene nanoplatelets.

The negligible oxygen content in the highly pure GANF is increased to around $38 \mathrm{wt} . \%$ in the functionalized nanoplatelets whereas the $\mathrm{C} / \mathrm{O}$ ratio in their surface is $\sim 3.8$ (Table S1). The analysis of C1s XPS spectra reports a somewhat higher density of $\mathrm{Csp}^{2}$ and $\mathrm{C}-\mathrm{O}$ (epoxy, hydroxyl) functionalities at the expense of $\mathrm{C}=\mathrm{O}$ (carbonyl) groups in OF1.

Nevertheless, XRD spectra (Fig. 3) clearly illustrate the impact of the oxidation time on the structure of the resulting oxygen-rich nanoplatelets. The intense crystalline (002) peak at $2 \theta=26.0^{\circ}$ observed for the highly graphitic nanofibers is shifted to a broad band centered at $23.5^{\circ}$ for OF1 (Fig. 3a) indicative of a disordered carbon structure with a somewhat larger interlayer spacing (centered at $\sim 3.8 \AA$ ). It appears that $150 \mathrm{~min}$ is not sufficient to succeed with the intercalation of functional groups.

On the other hand, oxidation (2) based on a longer treatment results in an efficient intercalation with subsequent structure expansion. The prominent (001) peak at $10.9^{\circ}$ in the OF2 pattern (Fig. 3b) reflects the enlargement of the basal spacing to $8.2 \AA$ due to functional groups randomly attached to the graphene layers. This value is in the range found for graphite oxide materials obtained by typical Hummers' procedure. ${ }^{30}$ 

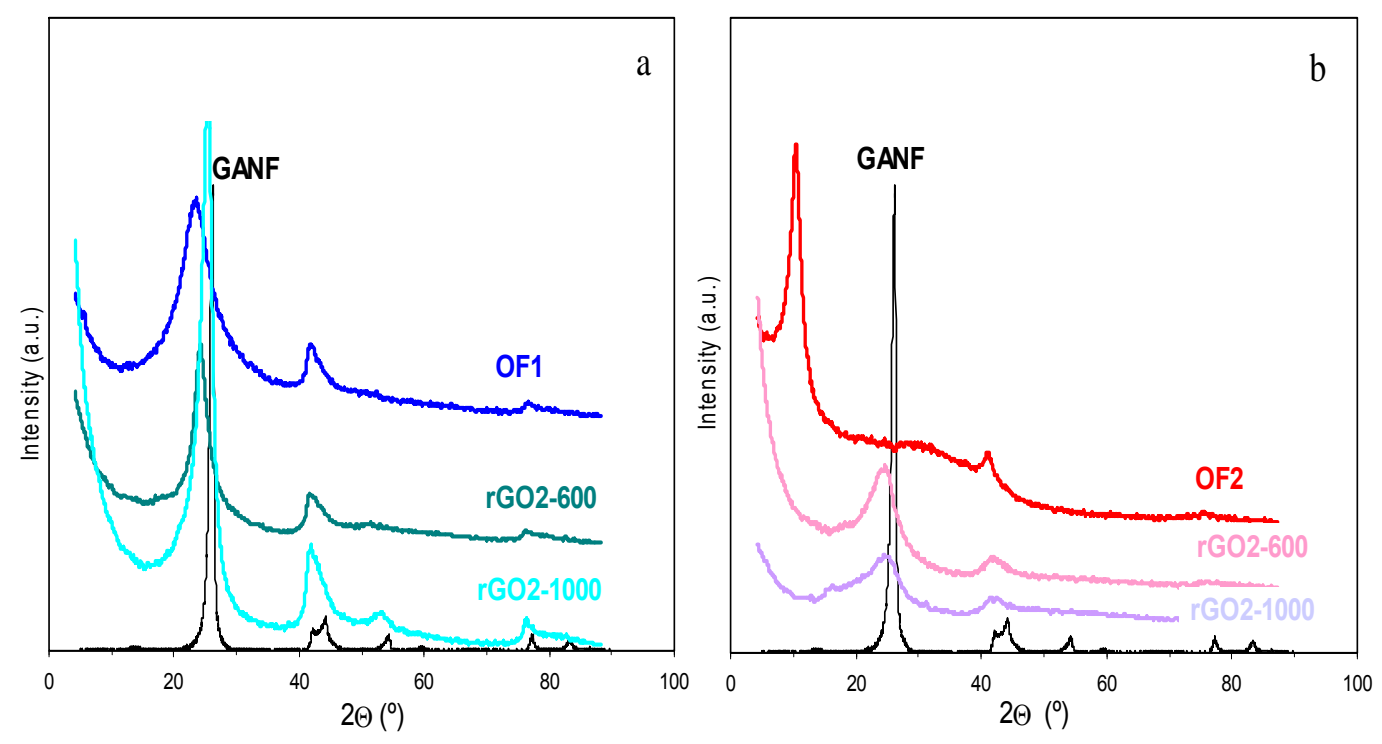

Fig. 3 XRD spectra of carbon nanofibers, functionalized graphene nanoplatelets and reduced graphene oxides.

TPD experiments confirm further differences in the functionalized graphene nanoplatelets. OF2 is thermally unstable and experiences a sudden deintercalation of O-groups as $\mathrm{CO}$ and $\mathrm{CO}_{2}$ at a temperature as low as $140^{\circ} \mathrm{C}$ (Fig. 2b). Such gases removal at high rate generates an internal pressure that exceeds the Van der Waals forces that hold the graphene sheets together and it results in blasting. ${ }^{3,31}$ The labile oxygen-containing functionalities desorbing at $\sim 80-200^{\circ} \mathrm{C}$ are less significant in OF1 and the $\mathrm{CO}_{2}$ and $\mathrm{CO}$ evolution takes place gradually over a wide temperature range. As a consequence of this progressive gas removal and of the less expanded structure, OF1 does not undergo blasting.

The $\mathrm{N}_{2}$ adsorption on GANF (Fig. 4a) reports a specific surface area of 107 $\mathrm{m}^{2} / \mathrm{g}$ which corresponds to the inner and outer surfaces of nanofibers with diameters from 20 to $80 \mathrm{~nm}^{13}$ Such structure disappears when the nanofibers are unraveled by oxidation (Fig. 5), the resulting porosity depending largely on the treatment duration. Whereas the $\mathrm{N}_{2}$-surface area of the agglomerated fluffy nanoplatelets obtained by oxidation (1) drops to $4 \mathrm{~m}^{2} / \mathrm{g}$, the longer process (2) yields bigger layered nanoplatelets (Fig. 5) with $45 \mathrm{~m}^{2} / \mathrm{g}$ (Fig. 4a). 

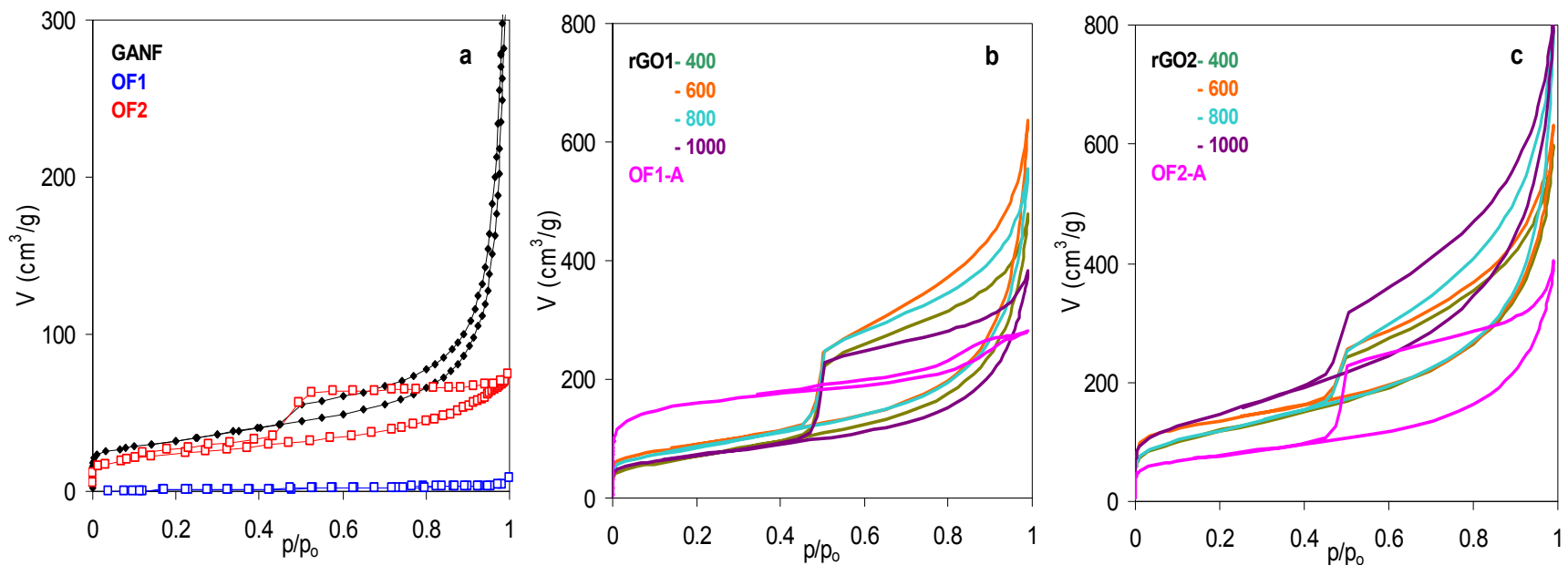

Fig. $4 \mathrm{~N}_{2}$ adsorption isotherms of carbon nanofibers, oxygen-rich graphene nanoplatelets, reduced graphene oxides and activated carbon nanoplatelets.

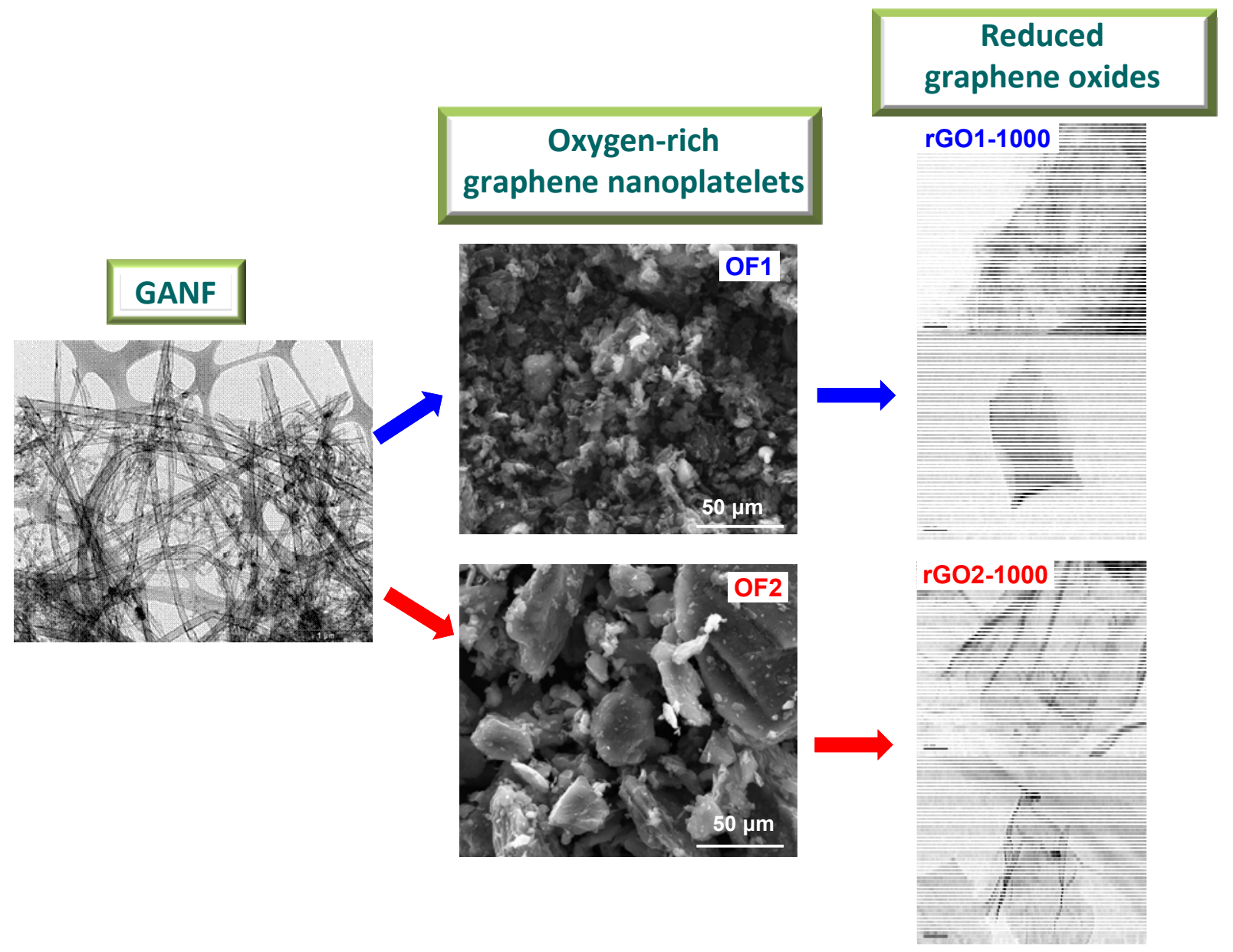

Fig. 5 SEM pictures of GANF and functionalized carbon nanoplatelets obtained by oxidation and TEM images of the reduced graphene oxides prepared by flash-pyrolysis at $1000^{\circ} \mathrm{C}$. 
TEM images of rGO1-1000 and rGO2-1000 (Fig. 5) illustrates the relevance of the interlayer spacing on the exfoliation success. In the case of the material obtained by oxidation overnight (OF2), a highly efficient exfoliation is achieved and the typical defected/wrinkled graphene sheets derived from thermal treatment of graphene oxides are obtained. On the contrary, the less expanded OF1 shows a limited exfoliation and separated graphene sheets are hardly detected in rGO1-1000 (Fig. 5).

The shape of the $\mathrm{N}_{2}$ isotherms of the rGOs (Figs. $4 \mathrm{~b}$ and $\mathrm{c}$ ) is typical type IV reported for exfoliated carbon fibers ${ }^{31}$ and reduced graphene oxides. ${ }^{32-34}$ They exhibit a well-defined capillary condensation step at $p / p_{o} \sim 0.5-0.8$ which reveals a wide mesopore size distribution. The hysteresis loop shows no limiting adsorption at high $\mathrm{p} / \mathrm{p}_{\mathrm{o}}$, which is often associated with slit-shaped pore coming from the aggregates of nanoplate-like particles. ${ }^{27}$ This effect is more significant for series derived from OF2 (Fig. 4c).

Fig. 6 illustrates that the evolution of the specific surface area of the rGOs with the flash-pyrolysis temperature is critically dependent on the precursor. For materials derived from OF1, the increase in the surface is virtually completed at around $400^{\circ} \mathrm{C}$, achieving a maximum value of $282 \mathrm{~m}^{2} / \mathrm{g}$ at $600^{\circ} \mathrm{C}$. On the other hand, the exfoliated materials from the more expanded nanoplatelets OF2 nearly duplicates the surface of those produced from OF1, reaching close to $500 \mathrm{~m}^{2} / \mathrm{g}$ at $1000^{\circ} \mathrm{C}$.

As a consequence of their different interlaying space, the functionalized nanoplatelets also demonstrated very distinctive behavior against standard activation process by $\mathrm{CO}_{2}$ at $800^{\circ} \mathrm{C}$. While such a treatment on OF1 generates a striking development of micropores, the activation of OF2 results in a mesoporous structure. Thus, OF1-A is an essentially microporous material with an average size of pores around $1.23 \mathrm{~nm}$ and very limited contribution from pores wider than $2 \mathrm{~nm}$ which reaches $389 \mathrm{~m}^{2} / \mathrm{g}$. This value is 1.4 times higher than that of the mesoporous rGO1-800 obtained by flash heating in air at the same temperature.

The activation of the more expanded OF2 leads to a limited increase of the surface. The blasting experienced at around $150^{\circ} \mathrm{C}$ appears to be the dominant process and OF2-A achieves only $250 \mathrm{~m}^{2} / \mathrm{g}$ which is half of the surface of the reduced graphene oxide rGO2-800. 
Regarding the surface chemistry, the treatment by $\mathrm{CO}_{2}$ produces a limited removal of functionalities and the oxygen content in OF1-A (15.0\%) and OF2-A $(12.8 \%)$ is, respectively, 50 and $30 \%$ higher than in the corresponding materials produced by flash pyrolysis at $800^{\circ} \mathrm{C}$.

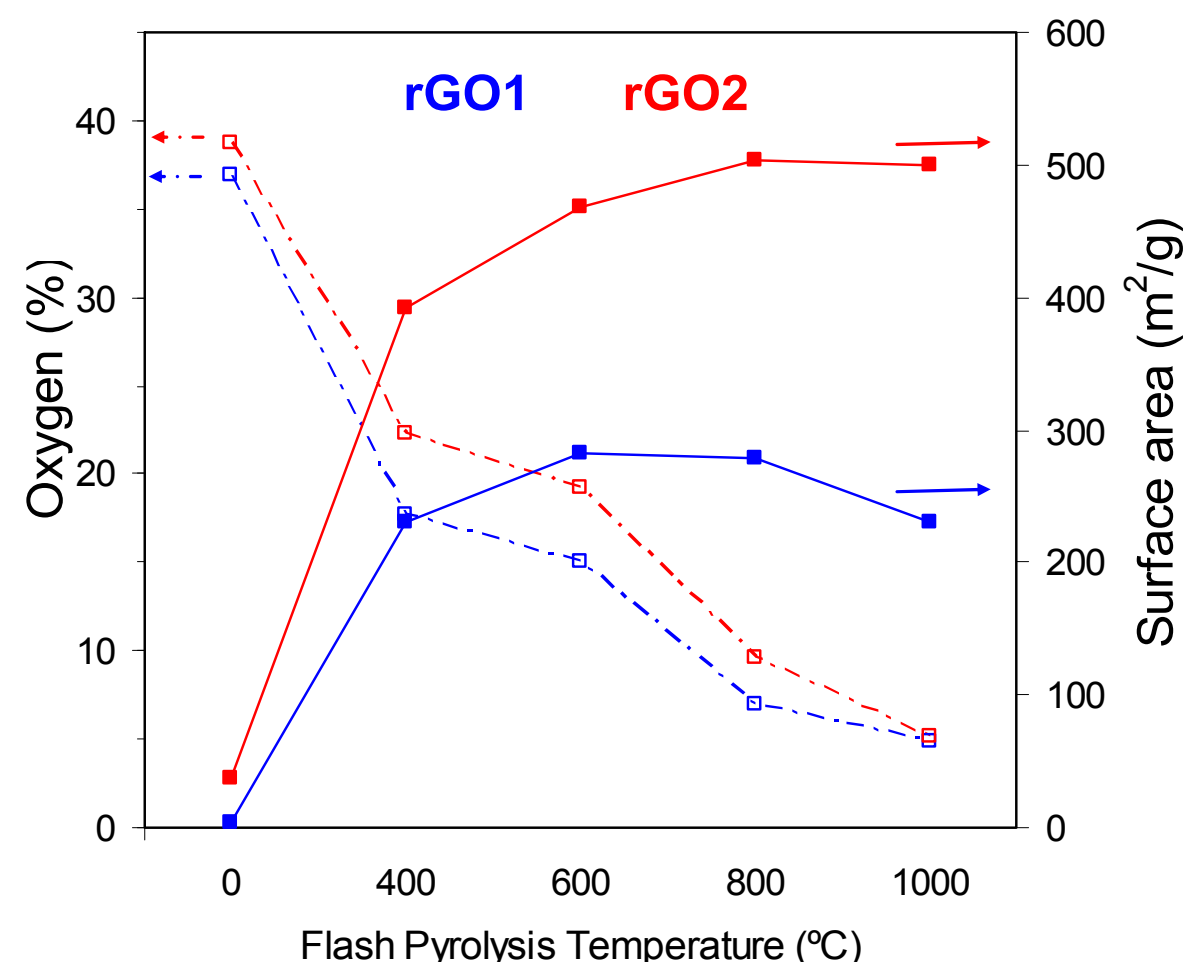

Fig. 6. Evolution of the specific surface area and oxygen content with flashpyrolysis temperature.

\section{Performance as supercapacitor electrodes}

The impact of the structural, textural and chemical changes experienced by the helical-ribbon GANF on the performance as SC electrodes can be simply monitored by cyclic voltammetry at different scan rates between 1 and $50 \mathrm{mV} / \mathrm{s}$. Similar patterns are obtained by galvanostatic charge-discharge cycles (Fig. S3) and confirm the different electrochemical operation of the diverse graphenerelated materials.

Fig. 7 briefly summarizes the general trends in the capacitance of the products generated by direct oxidation of carbon nanofibers and subsequent treatments (Fig. 1). Whereas the pristine carbon nanofibers GANF display only $10 \mathrm{~F} / \mathrm{g}$ at 1 $\mathrm{mV} / \mathrm{s}$, the capacitance of the functionalized nanoplatelets OF1 and OF2 greatly 
raises to, respectively, 82 and $104 \mathrm{~F} / \mathrm{g}$. It is impressive that these materials with only 4 and $45 \mathrm{~m}^{2} / \mathrm{g}$ can achieve nearly equal capacitance as that of activated carbons $\left(1000-1200 \mathrm{~m}^{2} / \mathrm{g}\right)$ used in commercial SC. ${ }^{34}$ As reported elsewhere, ${ }^{36}$ such outstanding surface-capacitance in $\mathrm{F} / \mathrm{m}^{2}$ only reflects the limitation of the standard $\mathrm{N}_{2}$ adsorption at $77 \mathrm{~K}$ to probe the effective surface involved in the ions adsorption in this type of carbons made of expanded carbon layers.

The comparison of the OFs performance illustrates the key role of their interlayer spacing. At low scan rate the capacitance of OF2 is significantly higher than that of OF1 (Fig. 7a) with clear contributions from redox reactions (broad peak at 0-0.5 V). It is likely that the more expanded structure of OF2 not only facilitates the ions access but also enhances their interaction with the oxygen-surface complexes on the carbon sheets. As a result, an extra pseudocapacitance involving surface oxygen-groups ${ }^{17,18,35}$ is added. In return, as the ions mobility is hindered by such slow redox processes, ${ }^{17}$ the capacitance of OF2 at $50 \mathrm{mV} / \mathrm{s}$ drops to about $25 \%$ of the value at $1 \mathrm{mV} / \mathrm{s}$ whereas OF1 maintains $50 \%$ of its limiting capacitance (Fig. $7 \mathrm{~b}$ ).

Noteworthy is the similar electrochemical behavior exhibited by the graphene oxides obtained by further high-energy ultrasonication of the oxidized nanoplatelets in water (Fig. 7c and d). At low current density, GO1 achieves $30 \%$ greater ability than that of the raw nanoplatelets whereas a slight variation is detected for GO2. This suggests that the exfoliation of OF1 allows access to such a large area as in the expanded material OF2. Despite this improvement, the separation of the carbon sheets and the presence of defects ${ }^{23}$ generate a resistive character in $\mathrm{GOs}^{3,18,33}$ and their capacitance is reduced by around 85$90 \%$ at $50 \mathrm{mV} / \mathrm{s}$ (Fig. $7 \mathrm{~d}$ ).

The arrangement of graphene sheets on the surface of the electrode may also play a role as far as they could align differently than the nanoflakes resulting in a lower charge effciciency ${ }^{24}$ under dynamic conditions. Therefore, their potential for supercapacitors is notably limited by the poor response rate. 
Functionalized Graphene Nanoplatelets
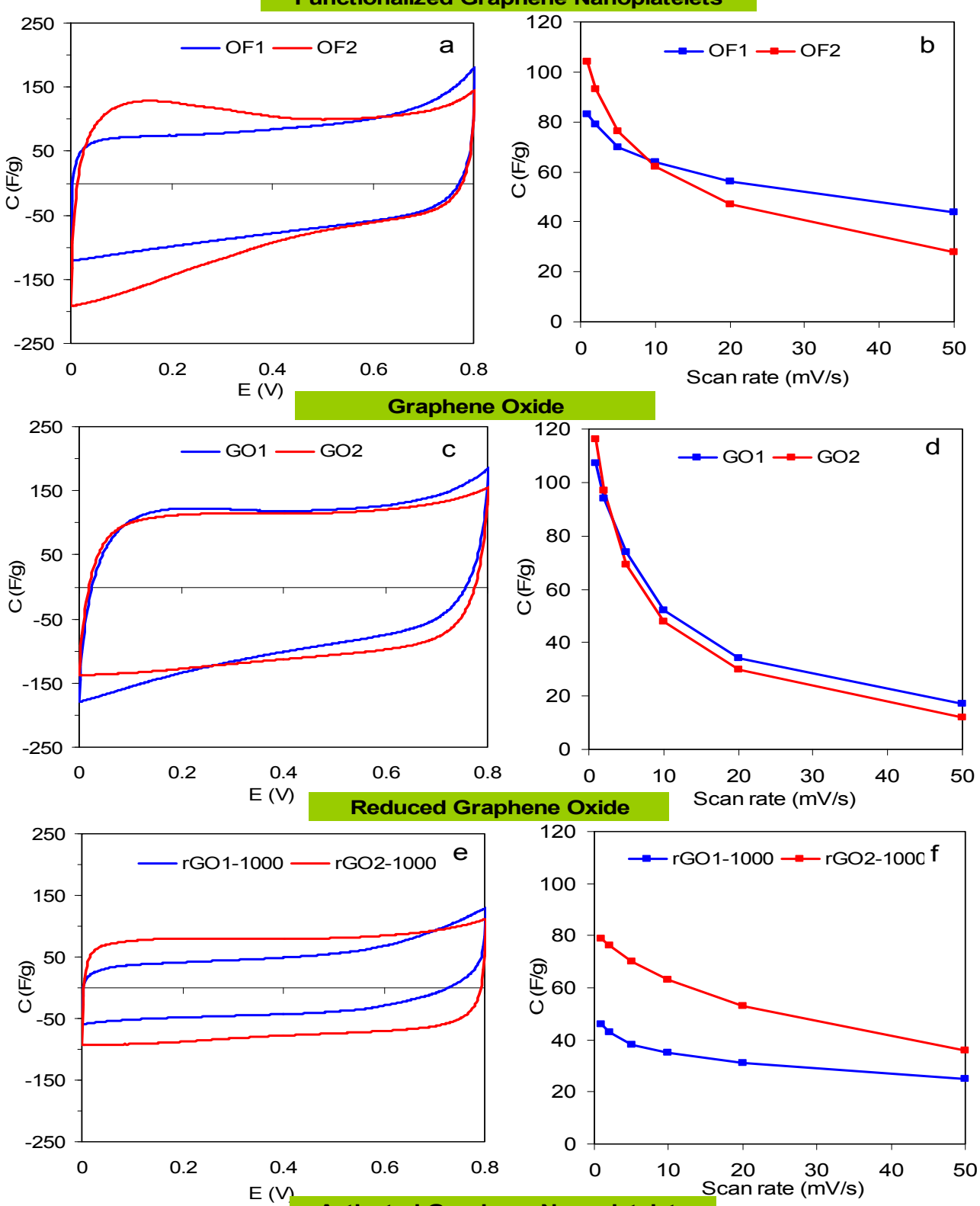

Activated Graphene Nanoplatelets

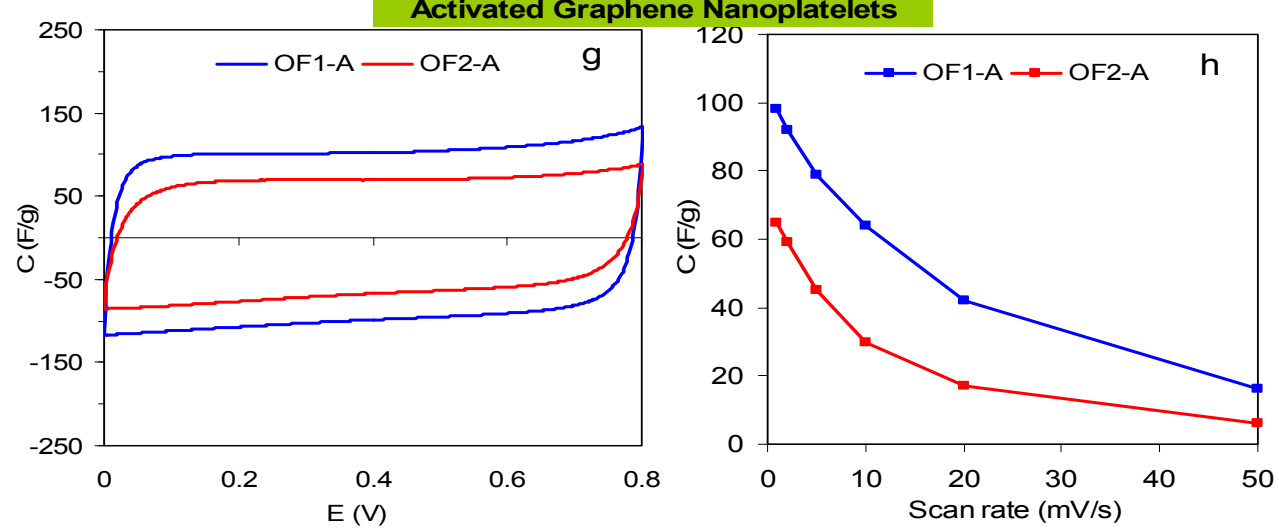

Fig. 7 Cyclic voltammograms at $1 \mathrm{mV} / \mathrm{s}(\mathrm{a}, \mathrm{c}, \mathrm{e}, \mathrm{g})$ and evolution of the specific capacitance with the scan rate $(b, d, f, h)$ for the different graphene-related materials. 
The removal of the oxygen functionalities by thermal processes leads to an enhancement of the electrical conductivity. ${ }^{3}$ As illustrated by the steeper current change at the switching potentials observed in the CV (Fig. 7e) and the symmetry and linear slopes of the charge-discharge cycles (Fig. S3), the rGOs obtained at $1000 \mathrm{C}$ display quicker charge propagation than the oxygen-rich nanoplatelets OFs and the graphene oxides GOs but the capacitance is much lower (Fig. 7f). They have only $5 \%$ of residual oxygen, six times lower than that of OFs and, therefore, the contribution from pseudocapacitance is much less significant. The reduced graphene oxides obtained at $1000^{\circ} \mathrm{C}$ follow the general pattern found for porous carbons with contributions of $0.16-0.17 \mathrm{~F} / \mathrm{m}^{2}$ typical for carbons with somewhat oxygen ${ }^{35,37}$ and essentially performing by double-layer formation. The larger specific surface area of rGO2-1000 helps to store more charges at the electrode-electrolyte interface and achieves $79 \mathrm{~F} / \mathrm{g}$ against 46 F/g of rGO1-1000.

By alternatively activating OFs with $\mathrm{CO}_{2}$ a very distinctive electrochemical behavior can be achieved. The microporous OF1-A doubles the limiting capacitance obtained by flash-pyrolysis at $1000^{\circ} \mathrm{C}$ although displays a lower effectiveness in terms of capacitance stability. In the case of the more expanded nanoplatelets OF2, this treatment gives a poor yield. The limiting capacitance is notably decreased to only $65 \mathrm{~F} / \mathrm{g}$ and around $90 \%$ is lost at 50 $\mathrm{mV} / \mathrm{s}$.

\section{Conclusions}

This study shows that wet oxidation of helical-ribbon carbon nanofibers (GANF) produced at industrial scale enables the production of a large variety of graphene-related materials.

By the control of the Hummers' based-oxidation time, unrevealing and cutting of GANF lead to highly functionalized graphene nanoplatelets with different interlaying spacing and, consequently, with a distinctive exfoliation potential. Their availability makes possible to obtain a number of graphenerelated products by ultrasonication or thermal treatments such as flash-pyrolysis in air at temperatures between 400 and $1000^{\circ} \mathrm{C}$ or standard activation with $\mathrm{CO}_{2}$ at $800^{\circ} \mathrm{C}$. The wide variety of structural, chemical and textural properties of the 
resulting graphene-like materials suggests their potential towards many applications.

A preliminary evaluation as supercapacitor electrodes in $2 \mathrm{M} \mathrm{H}_{2} \mathrm{SO}_{4}$ summarizes the impact of the changes experienced by the carbon nanofibers along the various processes. Whereas the helical-ribbon carbon nanofibers displayed only $10 \mathrm{~F} / \mathrm{g}$ in $2 \mathrm{M} \mathrm{H}_{2} \mathrm{SO}_{4}$, the functionalized nanoplatelets achieved a capacitance as high as $104 \mathrm{~F} / \mathrm{g}$. A more expanded structure not only facilitates the ions access but also enhances their interaction with the oxygen-surface complexes and nanoplatelets with larger spacing display an extra pseudocapacitance. By contrast, the ions mobility is hindered by slow redox processes and the corresponding supercapacitor shows a limited response rate.

Further exfoliation of oxygen-rich nanoplatelets leads to an increase in the specific capacitance up to $100-120 \mathrm{~F} / \mathrm{g}$ but the separation of the carbon sheets and the presence of defects generate a resistive character in the resulting graphene oxides.

The removal of the oxygen functionalities by flash-pyrolysis at $1000^{\circ} \mathrm{C}$ results in quicker charge propagation although the capacitance is much reduced due to less significant contributions from redox-type reactions.

The alternative activation of functionalized nanoplatelets with $\mathrm{CO}_{2}$ results to be more effective than flash heat treatment in air in terms of specific capacitance but does not succeed in capacitance stability.

It is worth noting that, among the present graphene-related materials, the lower-cost functionalized nanoplatelets displayed the better behavior for aqueous supercapacitor.

\section{Acknowledgements}

Financial support from EU 7FP (Project Electrograph- 266391) and EU FP7ICT-2013-FET-FGRAPHENE Flagship Project (Nr. 604391) is gratefully acknowledged. The authors thank the support of Servicios Científico-Técnicos (Edificio Severo Ochoa)-University of Oviedo and are especially grateful to Carlos Álvarez Villa for TEM images. 


\section{References}

1 W. Ren and H.-M. Cheng, Nature Nanotech., 2014, 9,726.

2 A. Zurutuza and C. Marinelli, Nature Nanotech, 2014, 9, 730.

3 Y. Zhu, S. Murali, W. Cai, X. Li, J.W. Suk, J.R. Potts and R.S. Ruoff, Adv. Mater., 2010, 22, 3906.

4 D.A.C. Brownson, D.K. Kampouris and C.E. Banks, J. Power Sources, 2011, 196, 4873.

5 D.A.C. Brownson and C.E. Banks, Analyst, 2010, 35, 2768.

6 Y.L. Zhong, Z. Tian, G.P. Simon and D. Li, Materials Today, 2015, 18, 73.

7 S.K. Sahoo and A. Mallik, Nano, 2015, 10, 1550019.

8 H.C. Schniepp, J.-L. Li, M.J. McAllister, H. Sai, M. Herrera-Alonso, D.H. Adamson, R.K. Prud'homme, R. Car, D.A. Saville and I.A. Aksay, J. Phys. Chem. B, 2006, 110, 8535.

9 S. Stankovich, D.A. Dikin, R.D. Piner, K.A. Kohlhaas, A. Kleinhammes, Y. Jia, Y. Wu, S.T. Nguyen and R.S. Ruoff, Carbon, 2007, 45, 1558.

10 D.V. Kosynkin, A.L. Higginbotham, A. Sinitskii, J.R. Lomeda, A. Dimiev, B.K. Price and J.M. Tour, Nature, 2009, 458, 872.

11 M. Terrones, Nature, 2009, 458, 845.

12 H. Varela-Rizo, I. Rodriguez-Pastor, C. Merino, M. Terrones and I. Martin-Gullón, J. Mater. Res., 2011, 26, 2632.

13 http://www.grupoantolin.com

14 J. Vera-Agullo, H. Varela-Rizo, J.A. Conesa, C. Almansa, C. Merino and I. Martin-Gullon, Carbon, 2007, 45, 2751.

15 M. Weisenberger, I. Martín-Gullon, J. Vera-Agullo, H. Varela-Rizo, C. Merino, R. Andrews, D. Qian and T. Rantell, Carbon, 2009, 47, 2211.

16 D. R. Bortz, E. Garcia Heras and I. Martin-Gullon, Macromolecules, 2012, 45, 238.

17 P. J. Hall, M. Mirzaeian, S. I. Fletcher, F. B. Sillars, A. J. R. Rennie, G. O. Shitta-Bey, G. Wilson, A. Cruden and R. Carter, Energy Environ. Sci., 2010, 3, 1238.

18 G. Wang, L. Zhang and J. Zhang, Chem. Soc. Rev., 2012, 41, 797.

19 Y. Huang, J. Liang and Y. Chen, Small, 2012, 8, 1805.

20 Y.B. Tan and J.-M. Lee, J. Mater Chem A, 2013, 1, 14814

21 Z. Liu, J. Liu, D. Li, P. S. Francis, N. W. Barnett, C. J. Barrowa and W. Yang, Chem. Commun., 2015, 51, 10969.

22 D.A.C. Brownson and C.E. Banks, Chem. Commun., 2012, 48, 1425.

23 W. Song, X. Ji, W. Deng, Q. Chen, C. Shena and C.E. Banks, Phys. Chem. Chem. Phys., 2013, 15,4799

24 W. Deng, X. Ji, M. Gómez-Mingot, F. Lu, Q. Chen and C. E. Banks, Chem. Commun., 2012, 48, 2770.

25 A. Ambrosi, S. Y. Chee, B. Khezri, R. D. Webster, Z. Sofer and M. Pumera, Angew. Chem., Int. Ed., 2012, 51, 500.

26 W.S.Hummers and R.E.Offeman, J. Am. Chem. Soc., 1958, 80, 1339.

27 K.S.W. Sing, Pure Appl. Chem., 1982, 54, 2201.

28 T.A. Centeno and F. Stoeckli, Carbon, 2010, 48, 2478.

29 M.D. Stoller and R.S. Ruoff, Energy Environ. Sci., 2010, 3, 1294.

30 H.P. Boehm, Angew. Chem. Int. Ed., 2010, 49, 9332.

31 M. Toyoda, Y. Tani and Y. Soneda, Carbon, 2004, 42, 2833.

32 H. Zhang, V.V. Bhat, N.C. Gallego and C.I. Contescu, ACS Appl. Mater. Interfaces, 2012, 4, 3239.

33 B. Xu, S. Yue, Z. Sui, X. Zhang, S. Hou, G. Cao and Y. Yang, Energy Environ. Sci., 2011, 4, 2826.

34 B. Lobato, V. Vretenar, P. Kotrusz, M. Hulman and T.A. Centeno, J. Colloid Interf. Sci., 2015, 446, 203.

35 M. Olivares-Marín, J.A. Fernández, M.J. Lázaro, C. Fernández-González, A. Macías-García, V. Gómez-Serrano, F. Stoeckli and T.A. Centeno, Mater. Chem. Phys., 2009, 114, 323.

36 B. Lobato, R. Wendelbo, V. Barranco and T. A. Centeno, Electrochim. Acta, 2014, 149, 245.

37 T.A. Centeno and F. Stoeckli, Electrochim. Acta, 2006, 52, 560. 